International Electronic Journal of Algebra

VOLUME 24 (2018) 153-173

DOI: $10.24330 /$ ieja.440239

\title{
A CATEGORICAL APPROACH TO ALGEBRAS AND COALGEBRAS
}

\author{
Robert Wisbauer \\ Received: 19 December 2017; Revised: 13 April 2018; Accepted: 14 April 2018 \\ Communicated by Abdullah Harmancı
}

Dedicated to the memory of Professor John Clark

\begin{abstract}
Algebraic and coalgebraic structures are often handled independently. In this survey we want to show that they both show up naturally when approaching them from a categorical point of view. Azumaya, Frobenius, separable, and Hopf algebras are obtained when both notions are combined. The starting point and guiding lines for this approach are given by adjoint pairs of functors and their elementary properties.
\end{abstract}

Mathematics Subject Classification (2010): 16H05, 16T05, 18C15, $18 \mathrm{C} 20$

Keywords: (Co)algebras, (co)monads, Frobenius algebras, separable algebras, Hopf algebras

\section{Introduction}

In the last decades categorical techniques turned out to be very effective in algebra and representation theory. Hereby, it was a key observation that module theory of an algebra $A$ over a field $K$ is essentially the theory of the functor

$$
A \otimes_{K}-: \mathbb{M}_{K} \rightarrow \mathbb{M}_{K}
$$

an endofunctor of the category of $K$-vector spaces. An algebra $A$ is defined by $K$-linear maps, multiplication $A \otimes_{K} A \rightarrow A$ and unit $e: K \rightarrow A$, subject to associativity and unitality conditions. Left $A$-modules are given by a $K$-vector space $V$ with $K$-linear maps $\varrho: A \otimes_{K} V \rightarrow V$, also subject to associativity and unitality conditions. Together with $A$-linear maps, this yields the category ${ }_{A} \mathbb{M}$ of left $A$-modules. The tensor product with multiplication $\left(A \otimes_{K} V, m \otimes_{K} V\right)$ is a left $A$-module and this leads to the free and forgetful functors

$$
\begin{aligned}
\phi_{A}: & \mathbb{M}_{K} \rightarrow{ }_{A} \mathbb{M}, V \mapsto\left(A \otimes_{K} V, m \otimes_{K} V\right), \\
U_{A}: & { }_{A} \mathbb{M} \rightarrow \mathbb{M}_{K},(M, \rho) \mapsto M,
\end{aligned}
$$


and the bijection

$$
\operatorname{Hom}_{A}\left(A \otimes_{K} V, M\right) \rightarrow \operatorname{Hom}_{K}\left(V, U_{A}(M)\right),
$$

that is, the functor $U_{A}$ is right adjoint to $A \otimes_{K}-$.

These basic structures can be defined for arbitrary categories $\mathbb{A}$, replacing $\mathbb{M}_{K}$, and any functor $F: \mathbb{A} \rightarrow \mathbb{A}$, replacing $A \otimes_{K}-: \mathbb{M}_{K} \rightarrow \mathbb{M}_{K}$. Multiplication and unit are replaced by natural transformations, $m: F F \rightarrow F$ and $\eta: 1 \rightarrow F$, satisfying the respective associativity and unitality conditions. This gives $F$ a monad structure.

An $F$-module is an object $V \in \mathbb{A}$ with a morphism $\varrho: F(V) \rightarrow V$ and for any $W \in \mathbb{A},\left(F(W), m_{W}\right)$ is an example for this. Morphisms of $F$-modules are morphisms from $\mathbb{A}$ respecting the module structures and they yield the category $\mathbb{A}_{F}$ of $F$-modules with free and forgetful functors

$$
\begin{array}{ll}
\phi_{F}: & \mathbb{A} \rightarrow \mathbb{A}_{F}, V \mapsto\left(F(V), m_{V}\right), \\
U_{F}: & \mathbb{A}_{F} \rightarrow \mathbb{A},(M, \varrho) \mapsto M,
\end{array}
$$

and the bijection

$$
\begin{aligned}
\operatorname{Mor}_{\mathbb{A}_{F}}(F(V), M) & \rightarrow \operatorname{Mor}_{\mathbb{A}}\left(V, U_{F}(M)\right), \\
F(V) \stackrel{f}{\rightarrow} M & \mapsto V \stackrel{\eta_{V}}{\rightarrow} F(V) \stackrel{f}{\rightarrow} M,
\end{aligned}
$$

shows that the functor $U_{F}$ is right adjoint to $\phi_{F}$.

Thus structures from module theory can be formulated in great generality. As we will see, if the functor $F: \mathbb{A} \rightarrow \mathbb{A}$ has a right adjoint $G: \mathbb{A} \rightarrow \mathbb{A}$, then the monad structure on $F$ provides $G$ with the structure of a comonad. This approach leads naturally to comonads (coalgebras, bocses) and comodules and we will highlight this interplay. Notice that the categorical tools developed are also successfully applied in theoretical computer science and logic (e.g. [26], [35]).

\section{Category theory}

The idea that the role of elements in algebraic structures should be taken over by homomorphisms came up from the beginning of the last century. It has finally been poured into a solid frame 1945 by Samuel Eilenberg and Saunders Mac Lane in the seminal paper [5]. For convenience, and to fix notation, we recall the basic notions and refer to [12] for more details.

2.1. Categories. A category $\mathbb{A}$ consists of a class of objects, and

(i) for each pair $\left(A, A^{\prime}\right)$ of objects, there is a set of morphisms $\operatorname{Mor}_{\mathbb{A}}\left(A, A^{\prime}\right)$, usually denoted by arrows $A \rightarrow A^{\prime}$, 
(ii) for any triple $A, A^{\prime}, A^{\prime \prime}$ of objects there is a composition of morphisms,

$$
\operatorname{Mor}_{\mathbb{A}}\left(A, A^{\prime}\right) \times \operatorname{Mor}_{\mathbb{A}}\left(A^{\prime}, A^{\prime \prime}\right) \rightarrow \operatorname{Mor}_{\mathbb{A}}\left(A, A^{\prime \prime}\right)
$$

which is associative in an obvious sense,

(iii) for any object $A, \operatorname{Mor}_{\mathbb{A}}(A, A)$ contains an identity morphism $1_{A}$, leaving any composition with it unchanged.

2.2. Functors. A covariant functor $F: \mathbb{A} \rightarrow \mathbb{B}$ between categories sends

(i) an object $A$ from $\mathbb{A}$ to an object $F(A)$ in $\mathbb{B}$,

(ii) a morphism $f: A \rightarrow A^{\prime}$ to $F(f): F(A) \rightarrow F\left(A^{\prime}\right)$ in $\mathbb{B}$,

(iii) a composit $f g$ of morphisms in $\mathbb{A}$ to $F(f g)=F(f) F(g)$ in $\mathbb{B}$,

(iv) the identity $1_{A}$ for $A$ in $\mathbb{A}$, to the identity of $F(A), F\left(1_{A}\right)=1_{F(A)}$.

It follows from this definition that $F$ induces a set map

$$
\Phi_{F}: \operatorname{Mor}_{\mathbb{A}}\left(A, A^{\prime}\right) \rightarrow \operatorname{Mor}_{\mathbb{B}}\left(F(A), F\left(A^{\prime}\right)\right),
$$

and $F$ is called faithful if $\Phi_{F}$ is injective, and full if $\Phi_{F}$ is surjective.

$F$ defines an equivalence of categories provided there exists a functor $G: \mathbb{B} \rightarrow \mathbb{A}$ such that $F G$ and $G F$ both are isomorphic to the respective identities.

A fully faithful functor $F: \mathbb{A} \rightarrow \mathbb{B}$ induces an equivalence between $\mathbb{A}$ and a full subcategory of $\mathbb{B}$, the image of $F$.

2.3. Natural transformations. Given two functors $F, G: \mathbb{A} \rightarrow \mathbb{B}$ between categories, a natural transformation $\psi: F \rightarrow G$ is given by a family of morphisms $\psi_{A}: F(A) \rightarrow G(A), A \in \mathbb{A}$, with commutative diagrams, for any morphism $h: A \rightarrow A^{\prime}$ in $\mathbb{A}$,

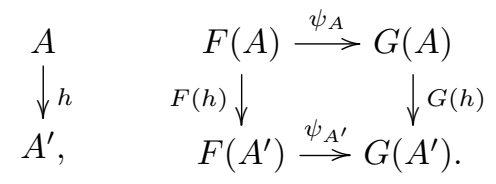

$\psi$ is called a (natural) isomorphism if all $\psi_{A}$ are isomorphisms in $\mathbb{B}$.

2.4. Separable functors. A functor $F: \mathbb{A} \rightarrow \mathbb{B}$ is said to be separable if, for any $A, A^{\prime} \in \mathbb{A}$, the canonical map $\Phi_{F}$ (from 2.2) is a naturally split monomorphism, that is, there is a map

$$
\Psi_{F}: \operatorname{Mor}_{\mathbb{B}}\left(F(A), F\left(A^{\prime}\right)\right) \rightarrow \operatorname{Mor}_{\mathbb{A}}\left(A, A^{\prime}\right),
$$

natural in $A, A^{\prime}$, with $\Psi_{F} \cdot \Phi_{F}$ the identity on $\operatorname{Mor}_{\mathbb{A}}\left(A, A^{\prime}\right)$. 
Clearly, for a separable functor $F, \Phi_{F}$ is always injective, and every fully faithful functor is separable. A survey on separability in algebra and category theory is given in [34].

It turned out that the following notion, formulated by D.M. Kan in [10] and further investigated by S. Eilenberg and J.C. Moore in [6] and others, is a milestone in category theory.

2.5. Adjoint pair of functors. A pair of (covariant) functors $F: \mathbb{A} \rightarrow \mathbb{B}$, $G: \mathbb{B} \rightarrow \mathbb{A}$ between any categories $\mathbb{A}, \mathbb{B}$, is said to be adjoint, we write $F \dashv G$, provided there is a bijection

$$
\alpha_{A, B}: \operatorname{Mor}_{\mathbb{B}}(F(A), B) \rightarrow \operatorname{Mor}_{\mathbb{A}}(A, G(B)),
$$

natural in $A \in \mathbb{A}$ and $B \in \mathbb{B}$. Such a bijection can be described by natural transformations, called unit and counit,

$$
\eta: 1 \rightarrow G F, \quad \varepsilon: F G \rightarrow 1,
$$

satisfying the triangular identities

$$
F \stackrel{F \eta}{\longrightarrow} F G F \stackrel{\varepsilon F}{\longrightarrow} F=1_{F}, \quad G \stackrel{\eta G}{\longrightarrow} G F G \stackrel{G \varepsilon}{\longrightarrow} G=1_{G} .
$$

They are obtained as images of the identities of $F(A)$ and $G(B)$, respectively, in the defining bijection.

Adjointness of contravariant functors $\mathbb{A} \rightarrow \mathbb{B}$ is defined by relating them with covariant functors between opposite categories.

2.6. Remark. The notion of adjointness can be weakened in various ways. For example, instead of being invertible one may require $\alpha$ to be regular, that is, there exists $\beta_{A, B}: \operatorname{Mor}_{\mathbb{A}}(A, G(B)) \rightarrow \operatorname{Mor}_{\mathbb{B}}(F(A), B)$, natural in $A \in \mathbb{A}$ and $B \in \mathbb{B}$, such that $\alpha \beta \alpha=\alpha$ (and $\beta \alpha \beta=\beta$ ). This yields a weaker form of the triangular identities (see [19], [32]).

A short argument shows that for adjoint functors $F \dashv G$,

- $F$ preserves epimorphisms and coproducts,

- $G$ preserves monomorphisms and products.

2.7. Properties of units and counits. Let $F \dashv G: \mathbb{B} \rightarrow \mathbb{A}$ be an adjoint pair of functors (notation from 2.5).

(1) $\varepsilon$ is an isomorphism if and only if $G$ is a fully faithful functor:

$G$ yields an equivalence between $\mathbb{B}$ and the image of $G$. 
(2) $\eta$ is an isomorphism if and only if $F$ is a fully faithful functor:

$F$ yields an equivalence between $\mathbb{A}$ and the image of $F$.

(3) $\varepsilon$ and $\eta$ are isomorphisms if and only if $F$ and $G$ both are fully faithful: $F$ and $G$ determine an equivalence between $\mathbb{A}$ and $\mathbb{B}$.

(4) $\varepsilon$ is a split epimorphism if and only if $G$ is a separable functor.

(5) $\eta$ is a split monomorphism if and only if $F$ is a separable functor.

2.8. Rings and modules. We follow the notation from [27]. For associative rings $R$ and $S$, denote by ${ }_{R} \mathbb{M}$ and $\mathbb{M}_{S}$ the category of left and right modules, respectively. Then any bimodule ${ }_{R} P_{S}$ induces an adjoint pair of functors

$$
{ }_{R} P \otimes_{S}-:{ }_{S} \mathbb{M} \rightarrow{ }_{R} \mathbb{M}, \quad \operatorname{Hom}_{R}(P,-):{ }_{R} \mathbb{M} \rightarrow{ }_{S} \mathbb{M}
$$

with bijection, counit, and unit

$$
\begin{gathered}
\operatorname{Hom}_{R}\left(P \otimes_{S} Y, X\right) \simeq \operatorname{Hom}_{S}\left(Y, \operatorname{Hom}_{R}(P, X)\right), \\
\varepsilon_{X}: P \otimes_{S} \operatorname{Hom}_{R}(P, X) \rightarrow X, \quad p \otimes f \mapsto f(p) \\
\eta_{Y}: Y \rightarrow \operatorname{Hom}_{R}\left(P, P \otimes_{S} Y\right), \quad y \mapsto[p \mapsto p \otimes y] .
\end{gathered}
$$

Denote by $\operatorname{Gen}(P)(\operatorname{Pres}(P))$ the category of $P$-generated ( $P$-presented) $R$ modules. Clearly the image of $P \otimes_{S}-$ is contained in $\operatorname{Pres}(P)$.

Choose an (injective) cogenerator $Q$ in ${ }_{R} \mathbb{M}$ and put $U=\operatorname{Hom}_{R}(P, Q)$. For the $S$-module $U$, denote by $\operatorname{Cog}(U)(\operatorname{Cop}(U))$ the category of $U$-cogenerated $(U$ copresented) $S$-modules. The image of $\operatorname{Hom}_{R}(P,-)$ is contained in $\operatorname{Cog}(U)$. If $R$ is a cogenerator in ${ }_{R} \mathbb{M}$ we can choose $U=P^{*}$.

(i) $\varepsilon$ isomorphism: $P$ is a generator in ${ }_{R} \mathbb{M}$ and the categories ${ }_{R} \mathbb{M}$ and $\operatorname{Cog}(U)$ are equivalent.

(ii) $\eta$ isomorphism: by 2.7, $P \otimes_{S}-$ is full and faithful and the categories ${ }_{S} \mathbb{M}$ and $\operatorname{Pres}(P)$ are equivalent (Sato equivalence, [23, Theorem 2.1]).

(iii) $\eta$ and $\varepsilon$ are isomorphisms: the categories ${ }_{R} \mathbb{M}$ and ${ }_{S} \mathbb{M}$ are equivalent; $P$ is a finitely generated projective generator in ${ }_{R} \mathbb{M}$ and $S=\operatorname{End}_{R}(P)$ (Morita equivalence, [21]).

(iv) $\varepsilon$ monomorph and $\eta$ epimorph: the categories $\operatorname{Gen}(P)$ and $\operatorname{Cog}(U)$ are equivalent (*-modules, e.g. [4], [24, Theorem 1.3], [28], [29]).

\section{Monads and comonads}

Monads and comonads on categories are modeled after the algebras and coalgebras on vector spaces. 
3.1. Monads and their modules. A monad on any category $\mathbb{A}$ is an endofunctor $T: \mathbb{A} \rightarrow \mathbb{A}$ with natural transformations, multiplication (product) and unit,

$$
m: T T \rightarrow T, \quad \eta: 1_{\mathbb{A}} \rightarrow T,
$$

subject to associativity and unitality conditions (as for algebras).

$T$-modules are objects $A \in \mathbb{A}$ with a morphism $\varrho: T(A) \rightarrow A$ subject to associativity and unitality conditions (as for modules over rings).

Morphisms between $T$-modules $(A, \varrho)$ and $\left(A^{\prime}, \varrho^{\prime}\right)$ (or T-morphisms) are morphisms $f: A \rightarrow A^{\prime}$ in $\mathbb{A}$ with commutative diagram

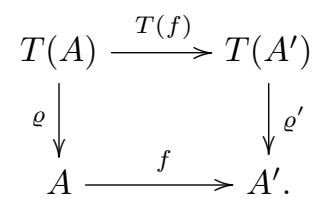

The category determined by $T$-modules and their morphisms is called the EilenbergMoore category - or just the module category - of the monad $(T, m, \eta)$ and we denote it by $\mathbb{A}_{T}$.

For any $A \in \mathbb{A}, T(A)$ has a $T$-module structure by $m_{A}: T T(A) \rightarrow T(A)$ and this leads to the free functor $\phi_{T}$ which allows for a right adjoint, the forgetful functor,

$$
\begin{aligned}
& \phi_{T}: \mathbb{A} \rightarrow \mathbb{A}_{T}, \quad A \mapsto\left(T(A), m_{A}\right), \\
& U_{T}: \mathbb{A}_{T} \rightarrow \mathbb{A}, \quad\left(A, \varrho_{A}\right) \mapsto A .
\end{aligned}
$$

The adjunction $\phi_{T} \dashv U_{T}$ is given by the bijection, for $A \in \mathbb{A}, B \in \mathbb{A}_{T}$,

$$
\operatorname{Mor}_{\mathbb{A}_{T}}\left(\phi_{T}(A), B\right) \stackrel{\simeq}{\rightarrow} \operatorname{Mor}_{\mathbb{A}}\left(A, U_{T}(B)\right), \quad f \mapsto f \eta_{A} .
$$

Putting $B=\phi_{T}\left(A^{\prime}\right)$ with $A^{\prime} \in \mathbb{A}$ and applying $U_{T} \phi_{T}=T$, we obtain

$$
\operatorname{Mor}_{\mathbb{A}_{T}}\left(\phi_{T}(A), \phi_{T}\left(A^{\prime}\right)\right) \stackrel{\simeq}{\rightarrow} \operatorname{Mor}_{\mathbb{A}}\left(A, T\left(A^{\prime}\right)\right) .
$$

Define a new category $\widetilde{\mathbb{A}}_{T}$ with the objects of $\mathbb{A}$ but choosing

$$
\operatorname{Mor}_{\widetilde{\mathbb{A}}_{T}}\left(A, A^{\prime}\right)=\operatorname{Mor}_{\mathbb{A}}\left(A, T\left(A^{\prime}\right)\right)
$$

and defining the composition of $f: A \rightarrow T\left(A^{\prime}\right)$ and $g: A^{\prime} \rightarrow T\left(A^{\prime \prime}\right)$ by

$$
g \diamond f: A \stackrel{f}{\longrightarrow} T\left(A^{\prime}\right) \stackrel{T(g)}{\longrightarrow} T T\left(A^{\prime \prime}\right) \stackrel{m_{A^{\prime \prime}}}{\longrightarrow} T\left(A^{\prime \prime}\right) .
$$

This is known as the Kleisli category of the monad $T$ and the bijection (3.1) shows that $\widetilde{\mathbb{A}}_{T}$ is isomorphic to the full subcategory of the Eilenberg-Moore category $\mathbb{A}_{T}$ determined by the objects $T(A), A \in \mathbb{A}$ (free $T$-modules).

Reversing the arrows in the definitions around monads yields 
3.2. Comonads and their comodules. A comonad on a category $\mathbb{A}$ is an endofunctor $S: \mathbb{A} \rightarrow \mathbb{A}$ with natural transformations, comultipliction (coproduct) and counit,

$$
\delta: S \rightarrow S S, \quad \varepsilon: S \rightarrow 1_{\mathbb{A}}
$$

subject to coassociativity and counitality conditions (dual to monad case).

$S$-comodules are objects $A \in \mathbb{A}$ with a morphism $\omega: A \rightarrow S(A)$ subject to coassociativity and counitality conditions.

Morphisms between comodules $(A, \omega)$ and $\left(A^{\prime}, \omega^{\prime}\right)$ (or $S$-morphisms), are morphisms $g: A \rightarrow A^{\prime}$ in $\mathbb{A}$ with commutative diagrams

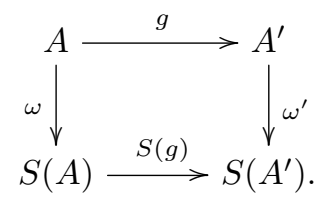

The category formed by the $S$-comodules and their morphisms is called the EilenbergMoore category - or just the comodule category - of the comonad $(S, \delta, \varepsilon)$ and we denote it by $\mathbb{M}^{S}$.

For any $A \in \mathbb{A}$, the structure map $\delta_{A}: S(A) \rightarrow S S(A)$ makes $S(A)$ an $S$ comodule. This yields the free functor $\phi^{S}$ which is right adjoint to the forgetful functor $U^{S}$,

$$
\begin{aligned}
& \phi^{S}: \mathbb{A} \rightarrow \mathbb{A}^{S}, A \mapsto\left(S(A), \delta_{A}\right), \\
& U^{S}: \mathbb{A}^{S} \rightarrow \mathbb{A},(A, \omega) \mapsto A .
\end{aligned}
$$

The adjunction $U^{S} \dashv \phi^{S}$ is given by the bijection, $B \in \mathbb{A}^{S}, A^{\prime} \in \mathbb{A}$,

$$
\operatorname{Mor}^{S}\left(B, S\left(A^{\prime}\right)\right) \stackrel{\simeq}{\rightarrow} \operatorname{Mor}_{\mathbb{A}}\left(U^{S}(B), A^{\prime}\right), \quad h \mapsto \varepsilon_{A^{\prime}} h .
$$

For $B=S(A)$ with $A \in \mathbb{A}$, using $U^{S} \phi^{S}=S$, we obtain

$$
\operatorname{Mor}^{S}\left(S(A), S\left(A^{\prime}\right)\right) \stackrel{\simeq}{\rightarrow} \operatorname{Mor}_{\mathbb{A}}\left(S(A), A^{\prime}\right) .
$$

Define a new category $\widetilde{\mathbb{A}}^{S}$ with the objects of $\mathbb{A}$ but choosing

$$
\operatorname{Mor}_{\widetilde{\mathbb{A}}^{S}}\left(A, A^{\prime}\right):=\operatorname{Mor}_{\mathbb{A}}\left(S(A), A^{\prime}\right)
$$

and take as composition, of $h: S(A) \rightarrow A^{\prime}$ and $k: S\left(A^{\prime}\right) \rightarrow A^{\prime \prime}$,

$$
k \diamond h: S(A) \stackrel{\delta_{A}}{\longrightarrow} S S(A) \stackrel{S(h)}{\longrightarrow} S\left(A^{\prime}\right) \stackrel{k}{\longrightarrow} A^{\prime \prime} .
$$

This is known as the Kleisli category of the comonad $S$. The bijection (3.4) shows that $\widetilde{\mathbb{A}}^{S}$ is isomorphic to the full subcategory of the Eilenberg-Moore category $\mathbb{A}^{S}$ determined by the objects $S(A), A \in \mathbb{A}$ ((co)free $S$-comodules). 
3.3. Rings and modules. For a ring $R$, any $(R, R)$-bimodule $A$ defines an endofunctor $A \otimes_{R}-:{ }_{R} \mathbb{M} \rightarrow{ }_{R} \mathbb{M}$. $A$ is called an $R$-ring if this functor allows for a monad structure. If $R$ is commutative and $r a=a r$ for $a \in A, r \in R$, an $R$-ring is called an $R$-algebra.

An $(R, R)$-bimodule $C$ is called an $R$-coring provided the functor $C \otimes_{R}-$ allows for a comonad structure. If $R$ is commutative and $r c=c r$ for $c \in C, r \in R$, an $R$-coring is called an $R$-coalgebra.

Notice that not every monad or comonad on ${ }_{R} \mathbb{M}$ can be represented by a tensor functor. For an extensive treatment of corings refer to [3].

\section{Adjoints and (co)monads}

The notion of adjoints and (co)monads are intimately related. Using naturality of the transformations involved it is straightforward to show:

4.1. From adjoints to (co)monads. Let $F \dashv G: \mathbb{B} \rightarrow \mathbb{A}$ be an adjoint pair of functors with unit $\eta: 1_{\mathbb{A}} \rightarrow G F$ and counit $\varepsilon: F G \rightarrow 1_{\mathbb{B}}$.

(i) $T:=G F: \mathbb{A} \rightarrow \mathbb{A}$ is an endofunctor and the natural transformations, multiplication and unit,

$$
m: T T=G F G F \stackrel{G \varepsilon F}{\longrightarrow} G F=T, \quad \eta: 1_{\mathbb{A}} \rightarrow G F=T,
$$

make $(T, m, \eta)$ a monad on $\mathbb{A}$.

(ii) $S:=F G: \mathbb{B} \rightarrow \mathbb{B}$ is an endofunctor and the natural transformations comultiplication and counit,

$$
\delta: S=F G \stackrel{F \eta G}{\longrightarrow} F G F G=S S, \quad \varepsilon: S=F G \rightarrow 1_{\mathbb{B}},
$$

make $(S, \delta, \varepsilon)$ a comonad on $\mathbb{B}$.

Recall that the construction of module and comodule categories in 3.1 and 3.2 show the inverse direction. In fact, these structures were introduced to show that

monads as well as comonads can be written as a composition of adjoint functors $([6],[11])$.

4.2. From (co)monads to adjoints. Let $\mathbb{A}$ be any category.

(i) For a monad $(T, m, \eta)$ on $\mathbb{A}$, the category $\mathbb{A}_{T}$ allows for an adjoint pair of functors $\phi_{T} \dashv U_{T}: \mathbb{A}_{T} \rightarrow \mathbb{A}$ with $T=U_{T} \phi_{T}$.

(ii) For a comonad $(S, \delta, \varepsilon)$ on $\mathbb{A}$, the category $\mathbb{A}^{S}$ allows for an adjoint pair of functors $U^{S} \dashv \phi^{S}: \mathbb{A} \rightarrow \mathbb{A}^{S}$ with $S=U^{S} \phi^{S}$. 
The same assertions hold replacing the Eilenberg-Moore categories by the corresponding Kleisli categories.

The structure of the (co)monads related to an adjunction are strongly influenced by the properties of the unit and counit.

4.3. Separability. Let $F \dashv G: \mathbb{B} \rightarrow \mathbb{A}$ be an adjunction as in 4.2 and $T=G F$, $S=F G$ the associated monad and comonad.

(i) Assume $G$ to be a separable functor (see 2.4). Then the monad $(T, m, \eta)$ allows for a comultiplication

$$
\delta^{\prime}: T=G F \stackrel{G \varepsilon^{\prime} F}{\longrightarrow} G F G F=T T,
$$

where $\varepsilon^{\prime}$ is a splitting for $\varepsilon$ (see 2.7(4)), with $m \cdot \delta^{\prime}=1_{T}$ and commutative diagrams (Frobenius conditions)
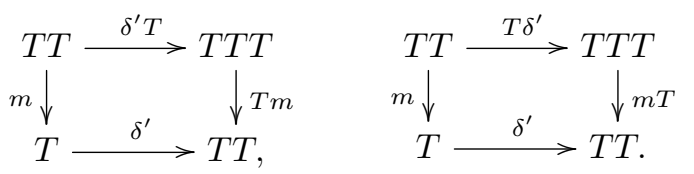

With these properties, $\left(T, m, \eta, \delta^{\prime}\right)$ is (called) a separable monad.

(ii) Assume $F$ to be a separable functor (see 2.4). Then the comonad $(S, \delta, \varepsilon)$ allows for a multiplication

$$
m^{\prime}: S S=F G F G \stackrel{F \eta^{\prime} G}{\longrightarrow} F G=S,
$$

where $\eta^{\prime}$ is a splitting for $\eta$ (see 2.7(5)), with $m^{\prime} \cdot \delta=1_{S}$ and commutative diagrams as in (4.1).

$\left(S, \delta, \varepsilon, m^{\prime}\right)$ is (called) a coseparable comonad.

4.4. Rings and modules. As in 2.8 , we consider an $(R, S)$-bimodule $P$. The related adjunction yields

$$
\begin{aligned}
\text { monad } & \operatorname{Hom}_{R}\left(P, P \otimes_{S}-\right):{ }_{S} \mathbb{M} \rightarrow{ }_{S} \mathbb{M}, \\
\text { comonad } & P \otimes_{S} \operatorname{Hom}_{R}(P,-):{ }_{R} \mathbb{M} \rightarrow{ }_{R} \mathbb{M} .
\end{aligned}
$$

Now $P^{*}:=\operatorname{Hom}_{R}(P, R)$ is an $(S, R)$-bimodule and hence induces

$$
\begin{aligned}
\operatorname{monad} & \operatorname{Hom}_{S}\left(P^{*}, P^{*} \otimes_{R}-\right):{ }_{R} \mathbb{M} \rightarrow{ }_{R} \mathbb{M} \\
\text { comonad } & P^{*} \otimes_{R} \operatorname{Hom}_{S}\left(P^{*},-\right):{ }_{S} \mathbb{M} \rightarrow{ }_{S} \mathbb{M} .
\end{aligned}
$$

If ${ }_{R} P$ is finitely generated and projective, $\operatorname{Hom}_{R}(P,-) \simeq P^{*} \otimes_{R}-$ and the comonad $P \otimes_{S} \operatorname{Hom}_{R}(P,-) \simeq P \otimes_{S} P^{*} \otimes_{R}-$, that is, $P \otimes_{S} P^{*}$ is an $R$-coring (and $P^{*} \otimes_{R} P \simeq S$ ). 
If $\eta$ is an isomorphism, $P \otimes_{S}$ - is (left) exact on the image of $\operatorname{Hom}_{R}(P,-)$ and hence the comonad

$$
P \otimes_{S} \operatorname{Hom}_{R}(P,-):{ }_{R} \mathbb{M} \rightarrow{ }_{R} \mathbb{M}
$$

is a left exact functor commuting with products in ${ }_{R} \mathbb{M}$. Therefore, by the dual of Watts' theorem (e.g. [9]), it is determined by the image of a cogenerator $Q \in{ }_{R} \mathbb{M}$, that is,

$$
\begin{aligned}
P \otimes_{S} \operatorname{Hom}_{R}(P,-) & \simeq \operatorname{Hom}_{R}\left(P \otimes_{S} \operatorname{Hom}_{R}(P, Q),-\right) \\
& \simeq \operatorname{Hom}_{S}\left(\operatorname{Hom}_{R}(P, Q), \operatorname{Hom}_{R}(P,-)\right) .
\end{aligned}
$$

In case $R$ is a cogenerator in ${ }_{R} \mathbb{M}$, we put $Q=R$ to obtain

$$
\begin{aligned}
P \otimes_{S} \operatorname{Hom}_{R}(P,-) & \simeq \operatorname{Hom}_{R}\left(P \otimes_{S} P^{*},-\right) \\
& \simeq \operatorname{Hom}_{S}\left(P^{*}, \operatorname{Hom}_{R}(P,-)\right) .
\end{aligned}
$$

This is a comonad, thus $P \otimes_{S} P^{*} \otimes_{R}$ - is a monad (by 5.1) which means that $P \otimes_{S} P^{*}$ is an $R$-ring (see 3.3 ).

If unit $\eta$ and counit $\varepsilon$ are isomorphisms, the comonad $P^{*} \otimes_{R} \operatorname{Hom}_{S}\left(P^{*},-\right) \simeq$ $P^{*} \otimes_{R} P \otimes_{S}-$, that is, $P^{*} \otimes_{R} P$ is an $S$-coring.

As a special case, we look at a situation studied in representation theory.

4.5. Nakayama functors. Let $A$ be a finite dimensional algebra over a field $K$ and $A$-mod the category of finitely generated left $A$-modules. It is customary to write $D(-)=\operatorname{Hom}_{K}(-, K)$, so $A^{*}=D(A)$, and one gets $\operatorname{Hom}_{K}(A,-) \simeq D(A) \otimes_{K}-$. The functor (e.g. [9])

$$
\nu=D \operatorname{Hom}_{A}\left(-,{ }_{A} A\right): A-\bmod \rightarrow A-\bmod
$$

is called the Nakayama functor, and

$$
\nu^{-}=\operatorname{Hom}_{A}\left(D(-), A_{A}\right): A-\bmod \rightarrow A-\bmod
$$

is said to be the inverse Nakayama functor. By the Eilenberg-Watts theorems one gets the natural isomorphisms

$$
\nu(-) \simeq D(A) \otimes_{A}-, \quad \nu^{-}(-) \simeq \operatorname{Hom}_{A}(D(A),-),
$$

thus there is an adjoint pair of endofunctors to be investigated.

From 2.8 and 4.4 one can see which monad or comonad structures may show up in this setting. The (A-module) structure of $D(A)$ is of course influenced by the structure of $A$. 


\section{Adjoint monads and comonads}

In 3.2 , comonads $(S, \delta, \varepsilon)$ were defined by reversing arrows in the definition of monads $(T, m, \eta)$. This does not mean that the two notions are strictly dual in a categorical way: in our definition we do not have anything like the "dual" of a functor $T$ ( or $S$ ). In this section we will outline that adjointness provides a bijective correspondence between monads and comonads.

5.1. Monads versus comonads Let $F \dashv G: \mathbb{A} \rightarrow \mathbb{A}$ be an adjoint pair of endofunctors with unit $\bar{\eta}$, counit $\bar{\varepsilon}$ and bijection, for $X, Y \in \mathbb{A}$,

$$
\alpha_{X, Y}: \operatorname{Mor}_{\mathbb{A}}(F(X), Y) \rightarrow \operatorname{Mor}_{\mathbb{A}}(X, G(Y)) .
$$

(i) A monad structure on $F$ induces a comonad structure on $G$, and vice versa, such that the associated (Eilenberg-Moore) categories $\mathbb{A}_{F}$ and $\mathbb{A}^{G}$ are isomorphic.

(ii) A comonad structure on $F$ induces a monad structure on $G$, and vice versa, such that the associated Kleisli categories $\widetilde{\mathbb{A}}_{F}$ and $\widetilde{\mathbb{A}}^{G}$ are equivalent.

Proof. (Sketch) (i) Let $(F, m, \eta)$ be a monad. The adjunction $F \dashv G$ induces the diagram

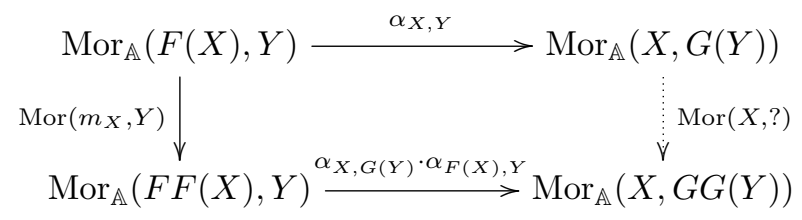

where the dotted arrow exists by applying the Yoneda Lemma to the composition of the other maps ( $\alpha$ is invertible) and determines a natural transformation $\delta: G \rightarrow$ $G G$. A similar argument shows the existence of a counit $\varepsilon: G \rightarrow 1_{\mathbb{A}}$. Explicitly we get

$$
\begin{gathered}
\delta: G \stackrel{\bar{\eta} G}{\longrightarrow} G F G \stackrel{G \bar{\eta} F G}{\longrightarrow} G G F F G \stackrel{G G m G}{\longrightarrow} G G F G \stackrel{G G \bar{\varepsilon}}{\longrightarrow} G G, \\
\varepsilon: G \stackrel{\eta G}{\longrightarrow} F G \stackrel{\bar{\varepsilon}}{\rightarrow} 1_{\mathbb{A}} .
\end{gathered}
$$

The symmetric construction shows that a comonad structure on $G$ leads to a monad structure on $F$. The equivalence of categories is given by

$$
\begin{aligned}
& \mathbb{A}_{F} \rightarrow \mathbb{A}^{G}, \quad F(A) \stackrel{h}{\longrightarrow} A \mapsto A \stackrel{\bar{\eta}_{A}}{\longrightarrow} G F(A) \stackrel{G(h)}{\longrightarrow} G(A), \\
& \mathbb{A}^{G} \rightarrow \mathbb{A}_{F}, \quad A \stackrel{\varrho}{\longrightarrow} G(A) \mapsto F(A) \stackrel{F(\varrho)}{\longrightarrow} F G(A) \stackrel{\bar{\varepsilon}_{A}}{\longrightarrow} A .
\end{aligned}
$$

(ii) By arguments symmetric to those in (i), a comonad structure on $F$ induces a monad structure on $G$, and vice versa. The equivalence of Kleisli categories $\widetilde{\mathbb{A}}^{F}$ 
and $\widetilde{\mathbb{A}}_{G}$ follows from the isomorphisms

$$
\begin{aligned}
\operatorname{Mor}_{\mathbb{A}^{F}}\left(\phi^{F}(A), \phi^{F}\left(A^{\prime}\right)\right) & \stackrel{(1)}{\simeq} \\
& \operatorname{Mor}_{\mathbb{A}}\left(F(A), A^{\prime}\right) \\
& \stackrel{(2)}{\simeq} \operatorname{Mor}_{\mathbb{A}}\left(A, G\left(A^{\prime}\right)\right) \\
& \stackrel{(3)}{\simeq} \operatorname{Mor}_{\mathbb{A}_{G}}\left(\phi_{G}(A), \phi_{G}\left(A^{\prime}\right)\right),
\end{aligned}
$$

where (1) is the isomorphism from (3.4), (2) is the adjunction, and (3) is the isomorphism from (3.2).

5.2. Frobenius monads. Let $F: \mathbb{A} \rightarrow \mathbb{A}$ be a self-adjoint endofunctor, that is, $F \dashv F$. By subsection 5.1, there are equivalent:

(a) F has a monad structure $(F, m, \eta)$;

(b) $F$ has a comonad structure $(F, \delta, \varepsilon)$.

With this (mutually) induced structures, $(F, m, \delta)$ satisfies the Frobenius conditions (4.1) and the categories $\mathbb{A}_{F}$ and $\mathbb{A}^{F}$ are isomorphic. These data define a Frobenius monad. ([18], [25]).

5.3. Rings and modules. Let $A$ be an $(R, R)$-bimodule and consider the adjoint functor pair of endofunctors (as in 2.8),

$$
A \otimes_{R}-, \operatorname{Hom}_{R}(A,-):{ }_{R} \mathbb{M} \rightarrow{ }_{R} \mathbb{M} .
$$

(i) An $R$-ring $(A, m, \eta)$, that is, a monad $A \otimes_{R}-$ on ${ }_{R} \mathbb{M}$, induces a comonad structure on $\operatorname{Hom}_{R}(A,-)$ with comultiplication

$$
\begin{aligned}
\operatorname{Hom}_{R}(A,-) \stackrel{\operatorname{Hom}(m,-)}{\longrightarrow} & \operatorname{Hom}_{R}\left(A \otimes_{R} A,-\right) \\
& \simeq \operatorname{Hom}_{R}\left(A, \operatorname{Hom}_{R}(A,-)\right),
\end{aligned}
$$

and the module category $\mathbb{M}_{A}$ is isomorphic to the comodule category $\mathbb{M}^{\operatorname{Hom}(A,-)}$.

(ii) A coring $(A, \delta, \varepsilon)$, that is, a comonad $A \otimes_{R}-$ on ${ }_{R} \mathbb{M}$, induces a monad structure on $\operatorname{Hom}_{R}(A,-)$ with multiplication

$$
\begin{array}{r}
\operatorname{Hom}_{R}\left(A, \operatorname{Hom}_{R}(A,-)\right) \simeq \\
\operatorname{Hom}_{R}\left(A \otimes_{R} A,-\right) \stackrel{\operatorname{Hom}(\delta,-)}{\longrightarrow} \operatorname{Hom}_{R}(A,-),
\end{array}
$$

and the Kleisli categories are isomorphic by assigning, for $X \in{ }_{R} \mathbb{M}$,

$$
\widetilde{\mathbb{M}}^{A} \rightarrow \widetilde{\mathbb{M}}_{\mathrm{Hom}(A,-)}, \quad A \otimes_{R} X \mapsto \operatorname{Hom}_{R}(A, X) .
$$

Note that the objects of $\mathbb{M}_{\mathrm{Hom}(A,-)}$ are also called contramodules (e.g. [2, Section 4], [31]). 
The $R$-ring $(A, m, \eta)$ is called a Frobenius $R$-ring provided $A \otimes_{R}$ - is a Frobenius monad on ${ }_{R} \mathbb{M}$. This implies that ${ }_{R} A$ is finitely generated and projective and hence $\operatorname{Hom}_{R}(A,-) \simeq A^{*} \otimes_{R}-$, thus $A \simeq A^{*}$ as left $A$-modules and $\mathbb{M}_{A} \simeq \mathbb{M}^{A}$. For commutative rings $R$ one obtains Frobenius algebras (as considered by $\mathrm{F}$. Frobenius in [7] over fields).

\section{Composition of monads and comonads}

Over a commutative ring $R$, the tensor product of two $R$-algebras $(A, m, e)$, $\left(B, m^{\prime}, e^{\prime}\right)$ can be made an algebra defining multiplication (writing $\otimes:=\otimes_{R}$ )

$$
\bar{m}_{A B}: A \otimes B \otimes A \otimes B \stackrel{A \otimes \mathrm{tw} \otimes B}{\longrightarrow} A \otimes A \otimes B \otimes B \stackrel{m \otimes m^{\prime}}{\longrightarrow} A \otimes B,
$$

with the twist map tw : $B \otimes A \rightarrow A \otimes B, b \otimes a \mapsto a \otimes b$.

This map is no longer available if $R$ is not commutative. On the other hand, the multiplication is formally defined replacing tw by any morphism $\tau: B \otimes A \rightarrow A \otimes B$, however, it is not clear which properties it has. So one may ask which conditions are to be satisfied by $\tau$ to make $\left(A \otimes B, \bar{m}_{A B}, e \otimes e^{\prime}\right)$ a unital algebra. These lead to commutative diagrams
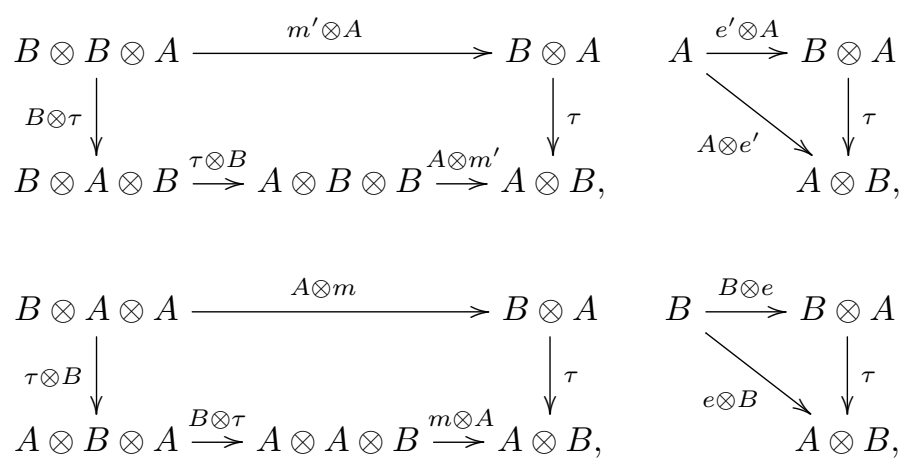

and should induce commutative diagrams of functors, with the forgetful functor $U_{B}:{ }_{B} \mathbb{M} \rightarrow{ }_{R} \mathbb{M}$,

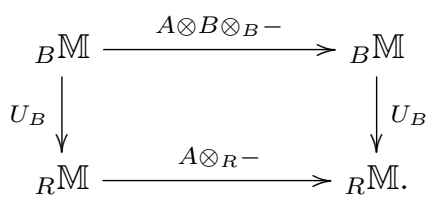

Then $A \otimes-\simeq A \otimes B \otimes \otimes_{B}-$ is called a lifting of $A \otimes-$ from ${ }_{R} \mathbb{M}$ to ${ }_{B} \mathbb{M}$.

The questions considered above for the functors $A \otimes-$ and $B \otimes-$ can be asked for endofunctors in any category. 
6.1. Liftings of endofunctors. Let $\mathbb{A}$ be any category with endofunctors $F, G$ : $\mathbb{A} \rightarrow \mathbb{A}$. If $(F, m, e)$ is a monad or $(G, \delta, \varepsilon)$ is a comonad, we get the two diagrams, respectively,
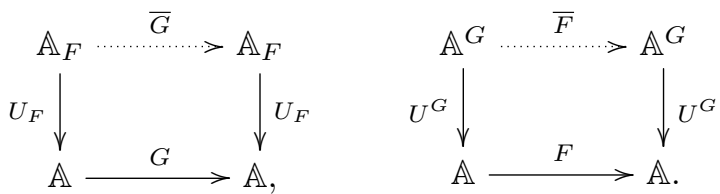

If $\bar{G}(\bar{F})$ exists it is called a lifting of $G(F)$ from $\mathbb{A}$ to $\mathbb{A}_{F}\left(\mathbb{A}^{G}\right)$. The following questions come up:

(i) when does a lifting $\bar{G}$ or $\bar{F}$ exist?

(ii) if $F$ and $G$ are monads, when is $\bar{G}$ a monad?

(iii) if $F$ and $G$ are comonads, when is $\bar{F}$ a monad?

(iv) if $F$ is a monad and $G$ is a comonad, when is $\bar{G}$ a comonad, when is $\bar{F}$ a monad?

All these problems can successfully be handled applying distributive laws as introduced and investigated in the 1970's by J. Beck [1] and others (see [30] for an overview and [2], [14], [15], et al. for more details).

Question (ii) above will lead to conditions which make the composition $F G$ a monad on $\mathbb{A}$ (as considered for algebras above) and (iii) describes the corresponding properties of comonads. Of particular interest are the questions in (iv) since they reveal an interesting interplay between monads and comonads.

6.2. Mixed distributive laws. Let $(F, m, \eta)$ be a monad and $(G, \delta, \varepsilon)$ a comonad on a category $\mathbb{A}$. A natural transformations $\lambda: F G \rightarrow G F$ is called a mixed distributive law or (mixed) entwining if it induces commutativity of the diagrams

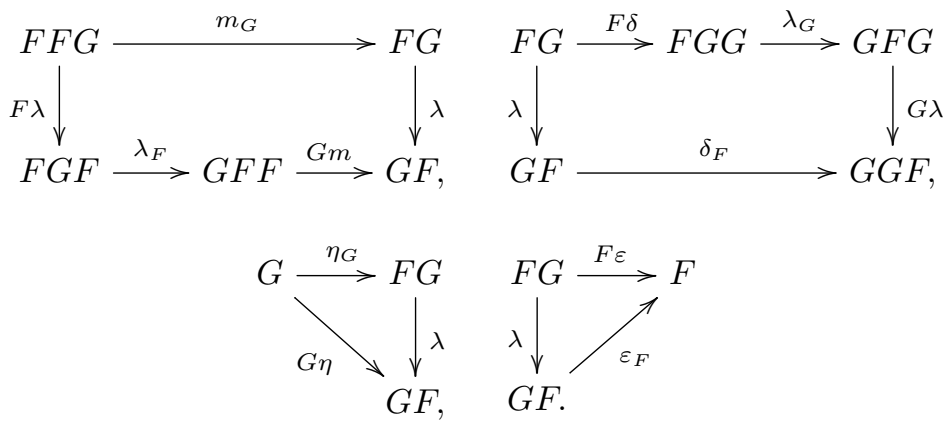

The following are equivalent (with notation from 6.1):

(a) $G$ can be lifted from $\mathbb{A}$ to $\bar{G}: \mathbb{A}_{F} \rightarrow \mathbb{A}_{F}$; 
(b) $F$ can be lifted from $\mathbb{A}$ to $\bar{F}: \mathbb{A}^{G} \rightarrow \mathbb{A}^{G}$;

(c) there exists a mixed distributive law $\lambda: F G \rightarrow G F$.

Given a monad and a comonad on $\mathbb{A}$, objects can have a module and a comodule structure. An entwining allows to require a compatibility of these structures.

6.3. Mixed modules. Let $(F, G, \lambda)$ be a mixed entwining and assume $A \in \mathbb{A}$ to be an $F$-module $\varrho: F(A) \rightarrow A$ and a $G$-comodule $\omega: A \rightarrow G(A)$. Then $(A, \varrho, \omega)$ is called a mixed $(F, G)$-module if we get commutativity of the diagram

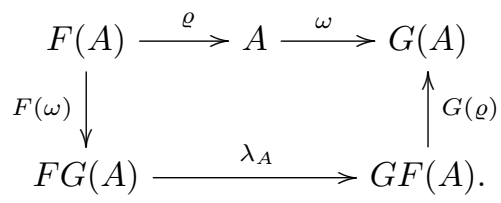

These objects with morphisms that are module as well as comodule morphisms, form a category which we denote by $\mathbb{A}_{F}^{G}$.

In 6.1, the lifting to Eilenberg-Moore categories was considered. A corresponding construction for Kleisli categories is the following.

6.4. Extending of endofunctors. Let $\mathbb{A}$ be any category with endofunctors $F, G: \mathbb{A} \rightarrow \mathbb{A}$. If $(F, m, e)$ is a monad or $(G, \delta, \varepsilon)$ is a comonad, we get the two diagrams (with $\phi$ the free functors), respectively,
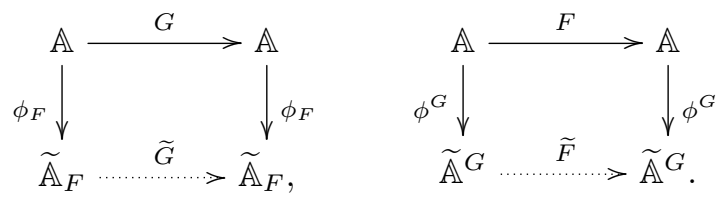

$\widetilde{G}$ and $\widetilde{F}$ are called the extensions of $F$ and $G$, respectively. Here again distributive laws apply for further investigation but with the role of monad and comonad interchanged, that is, one needs natural transformations $\sigma: G F \rightarrow F G$ inducing commutativity of the corresponding diagrams. Notice that the role of mixed modules (as in 6.3) does not transfer to this situation.

\section{Bimonad and Hopf monads}

Of special interest are endofunctors $B$ on $\mathbb{A}$ which carry a monad structure $(B, m, \eta)$ and a comonad structure $(B, \delta, \varepsilon)$ at the same time. To make these data a bimonad we first require the existence of a mixed distributive law $\lambda: B B \rightarrow B B$.

The relevant commutative diagrams are either for the monad structure or else for the comonad structure - they do not relate multiplication with comultiplication, 
for example. To connect these we need further compatibility conditions, namely commutativity of the diagrams
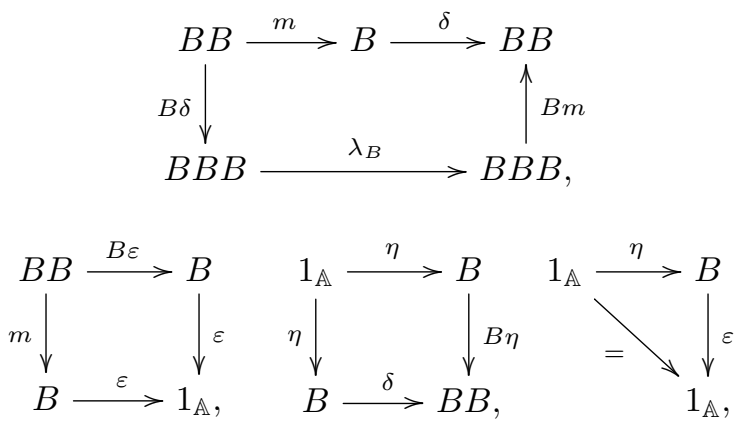

where the bottom diagrams mean that $\eta$ is comonad morphism and $\varepsilon$ a monad morphism. Diagram (7.1) guarantees that for each $A \in \mathbb{A}, B(A)$ is a mixed $(B, B)$ module in the sense of 6.3 , that is, $B(A) \in \mathbb{A}_{B}^{B}$. This determines a functor

$$
\phi_{B}^{B}: \mathbb{A} \rightarrow \mathbb{A}_{B}^{B}, \quad A \mapsto B B(A) \stackrel{m_{A}}{\longrightarrow} B(A) \stackrel{\delta_{A}}{\longrightarrow} B B(A)
$$

which is full and faithful by the isomorphisms (see 3.4, 3.1)

$$
\operatorname{Mor}_{B}^{B}\left(B(A), B\left(A^{\prime}\right)\right) \simeq \operatorname{Mor}_{B}\left(B(A), A^{\prime}\right) \simeq \operatorname{Mor}_{\mathbb{A}}\left(A, A^{\prime}\right) .
$$

A natural transformation $S: B \rightarrow B$ is called an antipode if it induces commutativity of the diagram

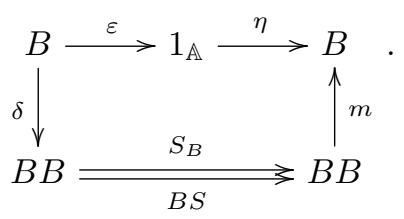

A bimonad which allows for an antipode is called a Hopf monad. Such an antipode exists if and only if either of the composites

$$
B B \stackrel{B \delta}{\longrightarrow} B B B \stackrel{m B}{\longrightarrow} B B, \quad B B \stackrel{\delta B}{\longrightarrow} B B B \stackrel{B m}{\longrightarrow} B B
$$

is an isomorphism $([16,5.5])$.

Under certain conditions on the category $\mathbb{A}$ and the functor $B$, the existence of an antipode $S$ is equivalent to $\phi_{B}^{B}: \mathbb{A} \rightarrow \mathbb{A}_{B}^{B}$ defining an equivalence $([16,5.6,6.11])$.

7.1. Adjoints of bimonads. Let $(B, m, \delta, \lambda)$ be a bimonad on $\mathbb{A}$ (as above). Assume $B$ allows for a right adjoint functor $C: \mathbb{A} \rightarrow \mathbb{A}$. Then by $5.1, C$ can be endowed with a comonad as well as a monad structure. Furthermore, there is a mixed distributive law $\lambda^{\prime}: C C \rightarrow C C$ (derived from $\lambda$, see $[16,7.4]$ ) which makes $C$ a bimonad. 
The bimonad $B$ has an antipode if and only if the associated bimonad $C$ has an antipode. Thus, given an adjoint pair $B \dashv C$ of functors on $\mathbb{A}, B$ allows for a Hopf monad structure if and only if so does $C$.

7.2. Bimonad on Set. ([30, 5.19], [16, 7.9]) For any set $G$, the cartesian product defines an endofunctor

$$
G \times-: \mathrm{SET} \rightarrow \mathrm{SET}, \quad A \mapsto G \times A,
$$

which is a comonad with comultiplication $\delta: G \rightarrow G \times G, g \mapsto(g, g)$, and is a monad provided $G$ is a monoid. Then it is a bimonad with entwining

$$
\lambda: G \times G \rightarrow G \times G, \quad(g, h) \mapsto(g h, g) .
$$

Now $G \times-$ has an antipode, i.e., is a Hopf monad, if and only if the monoid $G$ is in fact a group. By 7.1 , this is also equivalent to $\operatorname{Map}(G,-): \operatorname{SET} \rightarrow \operatorname{SET}$, a right adjoint of $G \times-$, being a Hopf monad.

7.3. Bialgebras. Let $R$ be a commutative ring. An $R$-module $B$ with an algebra structure $(B, m, \eta)$ and a coalgebra structure $(B, \delta, \varepsilon)$ is called a bialgebra if $\delta$ and $\varepsilon$ are algebra morphisms, or, equivalently, $m$ and $\eta$ are coalgebra morphisms. These conditions require commutativity of the outer path in the diagram

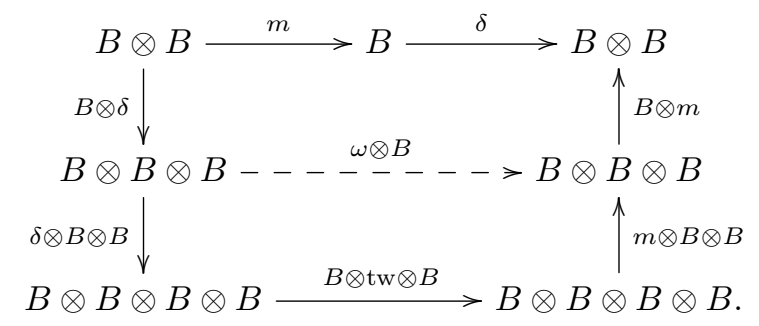

Defining an $R$-linear map

$$
\omega: B \otimes B \stackrel{\delta \otimes B}{\longrightarrow} B \otimes B \otimes B \stackrel{B \otimes \mathrm{tw}}{\longrightarrow} B \otimes B \stackrel{m \otimes B}{\longrightarrow} B \otimes B,
$$

the condition reduces to commutativity of the upper rectangle. As readily checked, $\omega: B \otimes B \rightarrow B \otimes B$ is a mixed distributive law between the monad $B \otimes-$ and the comonad $B \otimes-$. Commutativity of the upper rectangle is just the compatibility condition which makes $B$ a bialgebra. Thus a bialgebra $B$ corresponds to a monad $B \otimes-$ with the associated monad and comonad structures and the specific mixed distributive law $\omega$. The mixed $(B, B)$-modules $\mathbb{M}_{B}^{B}$ are called Hopf modules.

The composite

$$
\bar{\omega}: B \otimes B \stackrel{B \otimes \delta}{\longrightarrow} B \otimes B \otimes B \stackrel{\mathrm{tw} \otimes B}{\longrightarrow} B \otimes B \stackrel{B \otimes m}{\longrightarrow} B \otimes B
$$


yields a similar rectangle (sides interchanged). It gives a mixed distributive law between the related monad $-\otimes B$ and the comonad $-\otimes B$. It may also be considered as a mixed distributive law between the comonad $B \otimes-$ and the monad $B \otimes-$ (see $6.4)$.

An antipode for the bialgebra $B$ is an $R$-linear map $S: B \rightarrow B$ leading to commutativity of the diagram (7.3) for the monad $B \otimes-$.

Bialgebras $B$ with an antipode are called Hopf algebras and they are characterized by the fact that $\phi_{B}^{B}: \mathbb{M}_{R} \rightarrow \mathbb{M}_{B}^{B}$ is an equivalence, that is, every Hopf module is of the form $B \otimes X$, for some $X \in \mathbb{M}_{R}$ (Fundamental Theorem).

In case $B$ is a finite dimensional algebra over a field $K, B \otimes_{K}-$ is left adjoint to the endofunctor $\operatorname{Hom}_{K}(B,-) \simeq B^{*} \otimes_{K}-$, where $B^{*}=\operatorname{Hom}_{K}(B, K)$. Hence $B$ is a Hopf algebra if and only if $B^{*}$ is a Hopf algebra (see 7.1). This is known as the duality principle of Hopf algebras.

Hopf algebras were brought to light by Heinz Hopf in his seminal paper [8] in topology (1941). The algebraic essentials of this notion were extracted by MilnorMoore in [20] (1965). As a result, interest was also directed to the more elementary notions of coalgebras and corings as building blocks for the theory. It took several years until their value for representation theory was unveiled (1980) by A.V. Roiter in [22], there a coring is called bocs, and the interest is focused on Kleisli categories. In the meantime more attention is paid to coalgebraic aspects of finite dimensional algebras. For this we refer to the recent paper [13] by R. Marczinzik and the references given there.

7.4. Remark. As pointed out in subsection 7.3, the mixed distributive law for a bialgebra was derived from the canonical twist of the tensor product of modules over a commutative ring. This setting was extended to monoidal braided categories, where a general version of such a twist map is required. Concentrating on the properties needed, for endofunctors $B$ on any category it is sufficient to define a local braiding $\tau: B B \rightarrow B B$ to derive the corresponding theory. For details we refer to [16, Section 6$]$ and [17].

7.5. Rings and modules. The preceding sections show that comultiplication plays an important role in the structure theory of algebras. Summarising we consider an algebra $(A, m, \eta)$ over a commutative ring $R$ and assume $A$ to allow for a comultiplication $\delta: A \rightarrow A \otimes A$. Then $A$ becomes

(1) a separable algebra if $(A, m, \delta)$ satisfies the Frobenius condition and $m \cdot \delta=1_{A}$; 
(2) an Azumaya algebra if it is separable and $R \simeq C(A)$ (center of $A$ ); the category of $C(A)$-modules is equivalent to the category ${ }_{A} \mathbb{M}_{A}$ of $(A, A)$-bimodules;

(3) a Frobenius algebra if $(A, m, \delta)$ satisfies the Frobenius condition and $(A, \delta)$ has a counit $\varepsilon: A \rightarrow R$; every $A$-module has an $A$-comodule structure (Frobenius (bi)module, see [33]);

(4) a Hopf algebra if $(A, m, \delta)$ induce commutativity of $(7.1$ and $(7.2))$ and allows for an antipode; the category of $R$-modules is equivalent to the category $\mathbb{M}_{A}^{A}$ of mixed $(A, A)$-bimodules (e.g. [3]).

Acknowledgement. The author is grateful to Bachuki Mesablishvili for proofreading and helpful comments.

\section{References}

[1] J. Beck, Distributive laws, in Seminar on Triples and Categorical Homology Theory, B. Eckmann (ed.), Springer, Berlin, 80 (1969), 119-140.

[2] G. Böhm, T. Brzeziński and R. Wisbauer, Monads and comonads on module categories, J. Algebra, 322(5) (2009), 1719-1747.

[3] T. Brzeziński and R. Wisbauer, Corings and Comodules, London Mathematical Society Lecture Note Series, 309, Cambridge University Press, Cambridge, 2003.

[4] J. Clark and R. Wisbauer, Idempotent monads and $\star$-functors, J. Pure Appl. Algebra, 215(2) (2011), 145-153.

[5] S. Eilenberg and S. Mac Lane, General theory of natural equivalences, Trans. Amer. Math. Soc., 58(2) (1945), 231-294.

[6] S. Eilenberg and J. C. Moore, Adjoint functors and triples, Illinois. J. Math., 9 (1965), 381-398.

[7] F. Frobenius, Theorie der hypercomplexen Größen, Sitz. Kön. Preuss. Akad. Wiss., (1903), 504-537; Gesammelte Abhandlungen, art. 70, 284-317.

[8] H. Hopf, Über die Topologie der Gruppen-Mannigfaltigkeiten und ihre Verallgemeinerungen, Ann. of Math., 42(2) (1941), 22-52.

[9] S. O. Ivanov, Nakayama functors and Eilenberg-Watts theorems, J. Math. Sci., 183(5) (2012), 675-680.

[10] D. M. Kan, Adjoint functors, Trans. Amer. Math. Soc., 87 (1958), 294-329.

[11] H. Kleisli, Every standard construction is induced by a pair of adjoint functors, Proc. Amer. Math. Soc., 16 (1965), 544-546.

[12] S. Mac Lane, Categories for the Working Mathematician, 2nd edn, Graduate Texts in Mathematics, 5, Springer-Verlag, New York, 1998. 
[13] R. Marczinzik, A bocs theoretic characterization of gendo-symmetric algebras, J. Algebra, 470 (2017), 160-171.

[14] B. Mesablishvili, Entwining structures in monoidal categories, J. Algebra, 319(6) (2008), 2496-2517.

[15] B. Mesablishvili and R. Wisbauer, Galois functors and entwining structures, J. Algebra, 324(3) (2010), 464-506.

[16] B. Mesablishvili and R. Wisbauer, Bimonads and Hopf monads on categories, J. K-Theory, 7(2) (2011), 349-388.

[17] B. Mesablishvili and R. Wisbauer, Notes on bimonads and Hopf monads, Theory Appl. Categ., 26(10) (2012), 281-303.

[18] B. Mesablishvili and R. Wisbauer, QF functors and (co)monads, J. Algebra, 376 (2013), 101-122.

[19] B. Mesablishvili and R. Wisbauer, The fundamental theorem for weak braided bimonads, J. Algebra, 490 (2017), 55-103.

[20] J. W. Milnor and J. C. Moore, On the structure of Hopf algebras, Ann. of Math., 81(2) (1965), 211-264.

[21] K. Morita, Duality for modules and its applications to the theory of rings with minimum condition, Sci. Rep. Tokyo Kyoiku Daigaku Sect. A, 6 (1958), 83-142.

[22] A. V. Roiter, Matrix problems and representations of BOCSs, Representation Theory I, Lecture Notes in Math., 831, Springer, Berlin-New York, (1980), 288-324.

[23] M. Sato, Fuller's theorem on equivalences, J. Algebra, 52(1) (1978), 274-284.

[24] M. Sato, On equivalences between module subcategories, J. Algebra, 59(2) (1979), 412-420.

[25] R. Street, Frobenius monads and pseudomonoids, J. Math. Phys., 45(10) (2004), 3930-3948.

[26] D. Turi and G. Plotkin, Towards a mathematical operational semantics, Proceedings 12th Ann. IEEE Symp. on Logic in Computer Science, LICS'97, Warsaw, Poland, (1997).

[27] R. Wisbauer, Foundations of Module and Ring Theory, Algebra, Logic and Applications, 3, Gordon and Breach Science Publishers, Philadelphia, PA, 1991.

[28] R. Wisbauer, Tilting in module categories, Abelian groups, module theory, and topology (Padua, 1997), Lecture Notes in Pure and Appl. Math., 201, Dekker, New York, (1998), 421-444. 
[29] R. Wisbauer, Static modules and equivalences, Interactions between ring theory and representations of algebras (Murcia), Lecture Notes in Pure and Appl. Math., 210, Dekker, New York, (2000), 423-449.

[30] R. Wisbauer, Algebra versus coalgebras, Appl. Categ. Structures, 16 (2008), 255-295.

[31] R. Wisbauer, Comodules and contramodules, Glasg. Math. J., 52(A) (2010), 151-162.

[32] R. Wisbauer, Regular pairings of functors and weak (co)monads, Algebra Discrete Math., 15(1) (2013), 127-154.

[33] R. Wisbauer, Weak Frobenius monads and Frobenius bimodules, Algebra Discrete Math., 21(2) (2016), 287-308.

[34] R. Wisbauer, Separability in algebra and category theory, Proc. Aligarh, (2016).

[35] J. Worthington, A bialgebraic approach to automata and formal language theory, Ann. Pure Appl. Logic, 163(7) (2012), 745-762.

\section{Robert Wisbauer}

Department of Mathematics

University of Düsseldorf

Germany

e-mail: wisbauer@math.uni-duesseldorf.de 